Biaelectrochemistry and Bioenergetics 5, 625-634 (1978)

\title{
254 - Effect of the Adenine Moiety on the Electrochemical Behavior of Nicotinamide Adenine Dinucleotide; Possible Reduction of the Adenine
}

by Conrad O. Schmakel, Mark A. Jensen and Philip J. Elving The University of Michigan, Ann Arbor, Michigan ${ }_{4} 8$ rog, U.S.A.

Manuscript received May 3oth $197^{S}$

\section{Summary}

The presence of the adenine moiety very markedly affects the adsorption phenomena at the solution/electrode interface associated with nicotinamide adenine dinucleotide ( $\left.N+D^{\circ}\right)$ in aqueous media. Furthermore, a number of effects seen on the electrochemical reduction of $N_{A D}$ may be associated with faradaic reduction of the adenine. Such a reduction, which has been postulated as an initial step in the pulse radiolysis of $\mathrm{NAD}^{-}$, helps to rationalize the reversible reduction of adsorbed $\mathbf{N A D}^{-}$, where the ultimate reduction site, i.e., the pyridine ring, is separated from the solution/electrode interface by the adenine ring. The adenine moiety also participates in a redox couple with mercury.

\section{Introduction}

The pyridine nucleotide, nicotinamide adenine dinucleotide [NAD+ ; also known as diphosphopyridine nucleotide (DPN+) and coenzyme I] (Fig. I), is a major coenzyme for the class of enzymes known as dehydrogenases, which catälyze reversible redox processes in virtually all biological systems, involving transfer of hydrogen between substrate and coenzyme, e.g., yeast alcohol dehydrogenase (ADH) catalyzes oxidation of ethanol to acetaldehyde and the simultaneous reduction of $\mathrm{NAD}$ - to $\mathrm{r}, 4-\mathrm{NADH}$ via the effective net transfer of a hydride ion from the alcohol to the +-position of the pyridine ring.1,2 (In subsequent discussion, NADH is taken to mean $1,4-\mathbf{N A D H}$ ).

The gross reversible redox behavior of the NAD--NADH couple under physiological conditions has prompted study of the couple by polarographic technics. ${ }^{3-6}$ While many aspects of the electrochemical behavior of $\mathrm{NAD}^{+}, \mathrm{NADH}$, and model compounds have been thoroughly examined and explained, others have been only partially clarified or have not been considered. One such item is that of the possible reduction of the adenine moiety in the $\mathrm{NAD}^{-}$.

It is still not entirely clear what part the adenine moiety plays in the NAD redox pattern. Nuclear magnetic resonance, spectrophoto- 


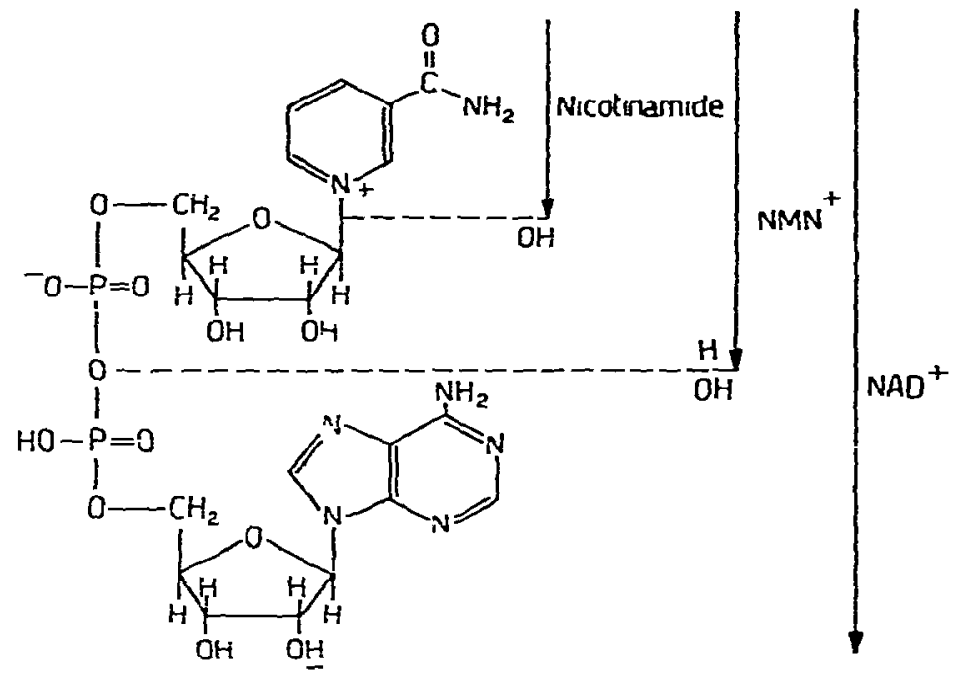

Fig. I.

Formula for enzymatacally active $\mathrm{VAO}^{-}$. 2 e., 3-nicotinamide adenine dinucleotide The somer having an $\alpha-$ glycosidic nicotinamide-ribose Inkage does not exhibit enz! matic activity with yeast $A D H$ A reiated coenzyme. nicatinamide adenine dinucleotide phosphate (NADPt). is formed when the underhned $H$ is replaced by a PO(OH)= group. In deamıno-NAD- (DNAD), the adenine morety is replaced by hypoxanthine

metric and fluorescence studies show considerable interaction between the nicotinamide and adenine moieties in solution with a large fraction of the dinucleotide being present in a folded conformation.7,8 A question is immediately raised as to whether the reducible species at the solution! electrode interface is folded or unfolded. Adenine could participate in the reduction with electrons received by it being transferred to the nicotinamide. The orientation of NAD- at the electrode surface is obviously of importance in this respect. In adenine-cytosine dinucleotides, both bases can be electrochemically reduced in the $\mathrm{pH}$ range of 2 to $5 .^{9}$

The present paper summarizes experimental observations on the electrochemical patterns seen with NAD- and reasonable deductions from such data, which indicate the distinct possibility that the adenine moiety may be reduced even though this may only result in a transitory reduced state due to, for example, transfer of the added electron or electrons from the adenine ring to the pyridine ring. It is hoped that the present account will stimulate more detailed investigation of the role plased by adenine in the electrochemical reduction of $\mathrm{NAD}^{+}$and the resulting implications for the behavior of the $N A D^{--N A D H}$ couple in biological systems.

The phenomena subsequently- discussed were seen in aqueous media unless otherwise specified. 


\section{Experimental}

Chemicals, apparatus and procedures, which are not subsequently discussed, have been described.10-15

The buffer solutions used, prepared from reagent grade chemicals, are listed in Table $I$.

Table r. Buffer and background electrolyte solutions $a$.

\begin{tabular}{|c|c|}
\hline pH Range & Composition \\
\hline $0.0-1.8$ & $\mathrm{HCl}+\mathbf{K C l}$ \\
\hline $2.0-8.0$ & $\begin{array}{l}\mathrm{Na}_{2} \mathrm{HPO}_{4}-7 \mathrm{H}_{2} \mathrm{O}+\text { citric } \\
\text { acid monohydrate }+\mathrm{KCl}\end{array}$ \\
\hline $3 \cdot 9-5 \cdot 9$ & HOAc + NaOAc \\
\hline $5+$ & HOAc + $\mathrm{NaOHc}+\mathrm{Et}_{4} \mathrm{NCl}^{b}$ \\
\hline 7.8 & $\mathrm{H}_{3} \mathrm{BO}_{3}+\mathrm{KCl}+\mathrm{NaOH}$ \\
\hline $90-$ ro.o & $\mathrm{K}_{2} \mathrm{CO}_{3}+\mathrm{KHCO}_{3}$ \\
\hline $9.0-10.0$ & $\mathrm{~K}_{2} \mathrm{CO}_{3}+\mathrm{KHCO}_{3}+\mathrm{KCl} c$ \\
\hline $90-10.0$ & $\mathrm{~K}_{2} \mathrm{CO}_{3}+\mathrm{KHCO}_{3}+\mathrm{Et}_{4} \mathrm{NCl} b$ \\
\hline $9.0-10.0$ & $\mathrm{NH}_{3}+\mathrm{NH}_{4} \mathrm{Cl}$ \\
\hline I I.0-I 2.0 & $\mathbf{K O H} \div \mathbf{K C I}$ \\
\hline
\end{tabular}

a The final ionic strength of buffer solutions in all experiments was $0.5 M$.

b $\mathrm{Et}_{4} \mathrm{NCl}$ concentration : $0.4 M$.

c $\mathrm{KCl}$ concentration : $0.4 \mathrm{Mr}$.

All potentials reported were measured with respect to the aqueous saturated calomel electrode (S.C.E.).

$\mathrm{NAD}^{+}$, unless otherwise indicated, refers to the normal biologically active $\beta$ form. Acronyms used to identify other compounds are as indicated : $\alpha-\mathrm{NAD}-(x$-nicotinamide adenine dinucleotide $), \mathbf{N A D P}+$ (nicotinamide adenine dinucleotide phosphate; triphosphopyridine nucleôtide; TPN- ; coenzyme II), DNAD+ (deamino-NAD ${ }^{-}$; nicotinamide hypoxanthine dinucleotide), DNADP - (deamino-NADP + ; nicotinamide hypoxanthine dinucleotide phosphate), AMP (adenosine- 5 -monophosphate); ADP (adenosine- $5{ }^{\prime}$-diphosphate), ATP (adenosine- 5 -triphosphate), NMN- (nicotinamide mononucleotide), NMNH (reduced nicotinamide mononucleotide), ADPR (adenosine diphosphate ribose).

\section{General polarographic patterns}

Adenine. - Adenine (6-aminopurine) is initially reduced electrolytically at a potential sufficient to reduce both the $r, 6$ and $3,2 N=C$ double bonds. On controlled potential clectrolysis, it undergoes a sixelectron $\left(6 \mathrm{e}^{-}\right)$reduction, which probably involves 
(a) $2 e^{-}$reduction of the $x, 6$ double bond to produce a gem-diamine centered on $\mathrm{C}(6)$,

(b) immediate $2 \mathrm{e}^{-}$reduction of this product to $x, 2,3,6$-tetrahy-droadenine,

(c) relativelr slow deamination at the 6-position to form 2,3 -dihydropurine,

(d) further $2 \mathrm{e}^{-}$reduction of the regenerated $\mathrm{r}, 6$ double bond form $1,2,3,6$-tetrahydropurine, and, finally,

(c) hydrolytic cleavage at the 2,3-position to form the same diazotizable amine as does purine in its overall $+c^{-}$reduction.9,16-1s

Under polarographic conditions at the dropping mercury electrode (D.M.E.), adenine exhibits one cathodic wave of the current magnitude expected for $a+e^{-}$wave, i.e., deamination does not proceed appreciably during the time scale involved in D.ML.E. polarography. The pH-dependence of the wave is given by

$$
C_{L_{3}}=-0.975-0.08_{4} \mathrm{pH}
$$

On crclic roltammetry at the hanging mercury drop clectrode (H.M.D.E.), adenine shows a single $\mathrm{pH}$-dependent cathodic peak; no complementary anodic peak is observed on the return scan even at fast scan rate. ${ }^{1 s}$ The peak current, $I_{p}$, is constant up to $\mathrm{pH} \mathbf{5} \cdot \mathbf{j}$ and then, similar to the D.M.E. Wave, decreases markedly with $\mathrm{pH}$ to vanish between pH 6 and 7 ; the wave is kinetically controlled in the $\mathrm{pH}$ region where it decreases. The latter effect is associated with the reducible form of adenine being the protonated species. In ammonia buffer, adenine gives an inflection close to the background discharge; point-by-point subtraction of the residual current rields a more or less well defined ware. ${ }^{27}$ Background discharge occurs at less negative potentials in the presence of adenine.

In aprotic media, adenine undergoes a $\mathrm{I} \mathrm{e}^{-}$reduction of $U_{y=}$ at $-2 .+\mathrm{r} \mathrm{V}^{r}$ in dimethy-l-formamide and dimethyl-sulfoxide, and at $-2.50 \mathrm{~V}$ in acetonitrile. ${ }^{19}$

NAD. - NAD-, similar to other I-substituted nicotinamides, shows two well-separated cathodic waves at the D.M.E. ${ }^{13}$ Interpretation of the behavior patterns is complicated by

(a) the presence of adsorption phenomena,

(b) potential shifts for wave Ic due to an irreversible dimerization following the initial reversible $I e^{-}$reduction,

(c) catalytic hydrogen evolution at lower $\mathrm{pH}$, and

(d) decomposition above $\mathrm{pH}$ ro, producing reducible nicotinamide.

Consequently, the characteristics of wares I $c$ and II $c$ can be studied thoroughly and simultaneously only between $\mathrm{pH} 9$ and ro. Over the $\mathrm{pH}$ range of 2 to 12 , $L_{e=}$ for wave $I c$ is $-0 . S 9 \perp 0.0 I V$ in the presence of background and buffer cations which are not more strongly adsorbed than $\mathrm{N}_{-} \mathrm{AD}^{-}$. Between $\mathrm{pH} 9$ and $\mathrm{ro}, C_{2}$ for wave II $c$ is $-\mathrm{I} .6+ \pm 0.03 \mathrm{~V}$; 
between $\mathrm{pH} 6$ and $S$, the wave does not appear to have a limiting plateau but gradually merges with solution discharge.

A small anodic wave is seen at times close to the potential of the mercury discharge.

Cyclic voltammetry indicates non-reduction of the wave I product in the wave II process and supports the notions that most of the NADT in the vicinity of the electrode forms the $I e^{-}$product before the potential for formation of the $2 \mathrm{e}^{-}$product is reached and that the peak II $c$ current is due to reduction of the $\mathrm{NAD}^{-}$, which diffuses through the depleted layer. At scan rates greater than $\mathrm{Io} V / \mathrm{s}$ and $\mathrm{pH}$ below 7 , a small cathodic peak $\left(U_{p}=-I .35 \mathrm{~V}\right)$ appears, which seems to grow with increasing scan rate; this peak may be due to adenine reduction and/or dimer desorption ( $c f$. subsequent discussion).

In aprotic media, $N^{\top} A D-$ gives a $I e^{-}$wave of $U_{\xi e}$ equal to - $0.98 \mathrm{~V}$; the second wate is not seen before background discharge at $-2.7 \mathrm{~V} .{ }^{20}$

\section{Effects of the adenine moiety in NADT}

The faradaic behavior of NAD- largely reflects the presence of the pyridine moiety and is, therefore, much the same as that, for example, of $\mathrm{NMN}+11$ (Fig. I) ; however, under certain conditions, the roltammetric patterns are more complicated as a result of adsorption and apparent faradaic phenomena due to the adenine moiety.

Adsorption phenomena. - The steps or wares that appear in the d.c. polarographic patterns of $N A D^{-}$at the D.M.E. in addition to the two $\mathrm{I} \mathrm{e}^{-}$reduction steps can be explained - largely on the basis of $a . c$. polarography - in terms of adsorption phenomena, e.g., adsorption of $\mathrm{NAD}^{+}$at the interface, replacing solvent molecules and background electrolyte and buffer species. In Et, NCl-carbonate buffer, a wave appears at approximately $-0.65 \mathrm{~V}$; the d.c. capacitive current is depressed before this wave but is essentially identical to that for the background electrolyte alone after the wave. An a.c. tensammetric peak at about the same potential is primarily capacitive in nature; the double layer capacitance is depressed before the peak, but, afterwards, is essentially identical to that for the background alone. The d.c. wave is, therefore, a capacitive step; before the step, $N A D^{-}$is adsorbed; after the step, Et $N_{-}$is preferentially adsorbed. The strong adsorption of the cationic $\mathrm{NAD}^{+}$on the positive side of the electrocapillary maximum (e.c.m.) results from the large tendency for adenine to be adsorbed even in its protonated form.9, ${ }^{91}$ On the negative potential side of the e.c.m., the surface-activity of the tetra-alkylammonium ion predominates.

In KCl-carbonate buffer, the d.c. polarographic pattern shows the capacitive current to be depressed over the entire potential region. A capacitive wave or maximum and an a.c. tensammetric peak, primarlycapacitive in nature, which appear at a potential intermediate between waves I and II $(c a$. - - I.3 V), has been ascribed to desorption of dimer; as the potential becomes more negative, the background electrolyte 
cation $\left(K^{-}\right)$displaces the dimer because of increasing coulombic attraction of $\mathrm{K}+$ to the electrode.

The fact that the adenine moiety plays a major role in the adsorption process can be seen by comparing the adsorptive behavior of NAD with NMN', which represents the nicotinamide portion of the NAD NMN - and its reduction products are negligibly adsorbed.11 NAD+ behaves similarly to nearly all adenine nucleosides and nucleotides in being strongly adsorbed in the potential region prior to and in the vicinity of the e.c.m., and in giving rise to desorption peaks at more negative potential.13,21 Similarly, the adenine or purine-related moiety of such NAD-related compounds as NADP $-, D^{-1} N^{-}, D_{N A D P}$, and $\alpha-N^{-} D^{+}$ causes adsorption behavior characteristic for each individual compound. On the addition of $\mathrm{Et}_{\mathrm{s}} \mathrm{NCl}$, adsorption of the dinucleotides beyond the e.c.m. is minimized due to the preferential adsorption ot $\mathrm{Et}_{4} \mathrm{~N}^{-}$; the d.c. polarographic patterns of all of the dinucleotides are then very similar to each other and to NMN-.

Mercury-adenine rompound formation. - D.M.E. polarograms of $\mathrm{NAD}+$ above $\mathrm{pH} g$ often exhibit, as mentioned, close to mercury discharge a small anodic wave, which also appears on a.c. polarography, e.g., the in-phase and quadrature current components are identical at low frequency $\left(\mathrm{I}_{5} \mathrm{~Hz}\right)$ but only the in-phase component appears at higher frequency (roo $\mathrm{Hz}$ ).

The electrode reaction can best be studied by cyclic voltammetry. Above $\mathrm{pH} 8$, a redox couple appears close to mercury discharge. In $\mathrm{pH}$ 9.3 carbonate buffer, the anodic and cathodic peaks of the couple are $20 \mathrm{mV}$ or less apart and of identical height after repetitive cycling at $0 . t V$ is in a potential region which includes both peaks. With increasing $\mathrm{pH}$, both peaks become more negative, i.e., the potential midway between them varies as follows:

$$
U=0.500-0.038 \mathrm{pH}
$$

The peaks increase in height with concentration but become independent of concentration above $0.04 \mathrm{~m} M$; even $0.001 \mathrm{~m} M \mathrm{NAD}-$ solutions yield peaks of constant height if the H.M.D.E. is placed in contact with the solution for a sufficient period of time before initiation of voltammetry. The constant peak heights indicate a process mainly dependent on clcctrode area. Similar results were found for AMP, ADP, ATP, and NADH, but not for nicotinamide or NMN-. NAD- did not give the redox couple at the pyrolytic graphite electrode.

These results indicate reaction of the adenine moiety with oxidized mercury to form a compound which coats the electrode surface ; the redox couple represents oxidation and reduction of mercury. Similar results have been reported for adenine itself. ${ }^{18}$ In the present study, a $\mathrm{pH} 4 . \mathrm{S}$ solution of ro $\mu M$ adenine showed this peak pair.

Possible adenine reduction. - The adenine moiety of $\mathrm{NAD}^{\mathrm{T}}$ and 
related compounds might be expected to behave similarly to such compounds as adenosine, AMP, ADP and ATP, whose electrochemical behavior resembles that of the parent adenine. ${ }^{16,21}$ Up to about $\mathrm{pH} 5$, these adenine-derived compounds generally exhibit a diffusion controlled $4 \mathrm{e}^{-}$ wave due to reduction of the protonated species; the wave is kineticallycontrolled at higher $\mathrm{pH}$ where it begins to disappear. $U_{1,}$ becomes more negative with increasing $\mathrm{pH}$. Solution discharge is more positive in their presence since adenine lowers the overpotential for hydrogen ion reduction even in slightly alkaline solution, where direct reduction is not seen, e.g., ADP in pH 8 MCIlvaine buffer shifts the background discharge roo $\mathrm{mV}$ positive.

Some evidence supporting electrolytic reduction of the adenine moiety in NAD - has been reported. ${ }^{22,23}$ Macroscale reduction of NAD in acidic medium at a large mercury pool cathode (at an uncontrolled potential of $-\mathrm{r}_{2} \mathrm{~V} v \mathrm{v}$. an isolated platinum anode) gave appreciable reduction of adenine, accompanied by an almost equivalent amount of deamination (under macroscale conditions, as previously noted, the initial $+\mathrm{e}^{-}$reduction product of adenine can be further reduced as a result of slow deamination at the 6-position regenerating the 1,6 double bond). At $\mathrm{pH} \mathrm{S}$, reduction of the adenine ring was sharply diminished and no deamination (as measured by ammonia erolution) was found.

On the basis of pulse radiolysis experiments, it was postulated that, when an electron reacts with the adenine moiety of $\mathrm{NAD}^{+}$, it transfers rapidly to the nicotinamide ring. ${ }^{24}$ Reduction by pulse radiolysis generally involves only a single electron, e.g., NAD+ forms a dimer. On electrolysis of $\mathrm{NAD}^{-}$in acidic solution at the H.M.D.E., virtually all of the $\mathrm{NAD}^{+}$in the vicinity of the electrode is converted to the dimer at a potential considerably more positive than the reduction potential of the adenine moiety; thus, no transfer of electrons from the latter to the oxidized pyridine moiety would be expected.

In the present study, following well-formed NAD+D.M.E. wave Ia $\left(U_{3, z}=-0.89 \mathrm{~V}\right)$, an ill-defined wave $\left(U_{1 / 2}=-\mathrm{r} .3 \mathrm{~V}\right)$ appears on the rising portion of solution discharge in $\mathrm{pH} 5$ acetate buffer (discharge is $25^{\circ}$ $\mathrm{mV}$ more positive in the presence of $\mathrm{NAD}^{+}$) ; under the same conditions, $\mathrm{NMN}^{+}$does not exhibit the latter wave, which is best studied at low temperature $\left(0 . \mathrm{I}^{\circ} \mathrm{C}\right.$ ) in low capacity acetate buffers between $\mathrm{pH} 5$ and 6 . At $\mathrm{PH} 5.6$, the limiting portion is virtually independent of mercury column height, but is strongly dependent on buffer capacity and NAD:concentration. In general, it is difficult to study the wave because of its sensitivity to experimental conditions.

In $0.5 M$ McIlvaine buffer of $\mathrm{pH}+.8$, o.I $\mathrm{m} M \mathrm{NAD}$ - shows the same D.M.E. polarographic wave at $-\mathrm{I} .37 \mathrm{~V}$ that ro $\mu M$ adenine shows in the same potential region (-I.35 to $-\mathrm{I} .37 \mathrm{~V})$ except that the wave height is approximately ten times that of the adenine wave.

On cyclic voltammetry of $\mathrm{NAD}^{-}$at $\mathrm{pH} 5.5$, an oddly sharp peak appears at - I-36 V on the rising background discharge; NMN+does not exhibit a similar peak (Fig. 2). Under similar conditions, only the peak corresponding to the first $\mathrm{I}^{-}$addition to $\mathrm{NAD}^{+}$has been reported. ${ }^{25}$ 
The potential and current of the peak seen at $-\mathrm{I} .36 \mathrm{~V}$ in acetate buffer depend on $\mathrm{pH}$ (Fig. 3) :

$$
U_{p}=-\mathbf{I . 0 7 t}-0.057 \mathrm{pH}
$$

The current, which decreases from five times that od $\mathrm{NAD}^{-}$peak Ic at $\mathrm{pH} 2$ to zero at $\mathrm{pH} 6.5$ and above, may involve a multiplicity of processes : direct adenine reduction, reduction of the pyridine moiety, catalytic hydrogen reduction promoted by adenine and the adenine and pyridine moiety reduction products, and sudden desorption of dimer, which might account for the sharpness of the peak. For example, the peak may be due to catalytic hydrogen evolution resulting from a rearrangement of the electrode double-layer during desorption, and/or reduction of unreduced $\mathrm{NAD}^{-}$in the vicinity of the electrode as the dimer film on the latter breaks. At high scan rates $(60 \mathrm{~V} / \mathrm{s})$, the NADreduction appears as a broad peak due to overlapping of the two peaks at -I.I and -I.3 V.

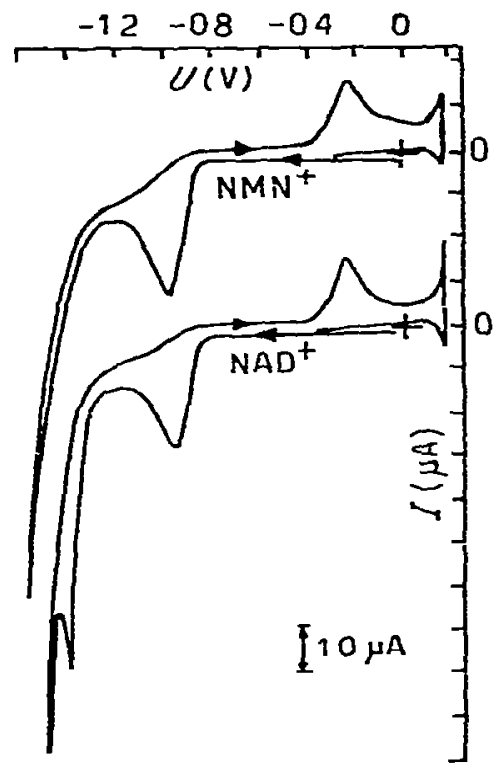

Fig. 2

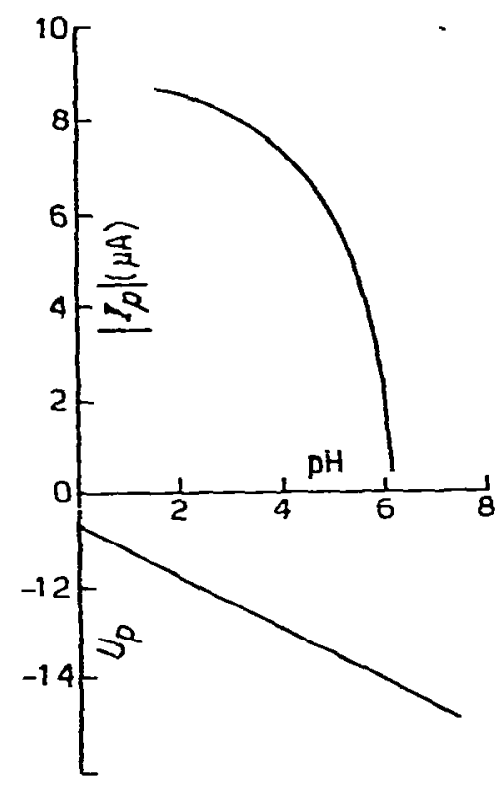

Fig. 3

Fig. 2.

Cy clic voltammograms at the $\mathrm{H} M \mathrm{M} \mathrm{E}$. of $\mathrm{NAD}^{+}(0+9 ., I)$ and NiI rate $=0 \mathrm{I} \mathrm{V} / \mathrm{s} \quad$ Electrode area $=0.029 \mathrm{~cm}^{2}$

Fig. 3

Variation in the voltammetric behas ior at the H MI D E with $\mathrm{pH}$ of the cathodic peak due to possible reduction of the $\mathrm{NAD}$ adenine molety. MICILVAINE and acetate buffers. NAD concentration $=032 \mathrm{~m} .1 /$ : electrode area $=0.3 \mathrm{r} \mathrm{cm}^{2}$. 
NADP - and $\alpha-N A D-$ behave similarly, except that the $-1.3 \mathrm{~V}$ peak disappears at $\mathrm{pH} 5$ for NADP+ and at $\mathrm{pH} 7$ for $\alpha-\mathrm{NAD}^{+}$. The peak is absent with NMN-, DNAD- and DNADP+, which do not contain the adenine moiety; the latter two compounds are more strongly adsorbed than $\mathrm{NAD}^{+}$. In $\mathrm{DNAD}^{+}$and $\mathrm{DNAP}^{-}$, reduction of the hypoxanthine moiety would be expected to be a $2 \mathrm{e}^{-}$process similar to that of hypoxanthine itself, which appears as an ill-defined inflection on background discharge. ${ }^{16}$

At higher frequencies (500-1000 $\mathrm{Hz}$ ) on a.c. polarography of $\mathrm{NAD}^{*}$, a faradaic peak $\left(U_{s}=-\mathrm{I} .32 \mathrm{~V}\right)$ is observed below $\mathrm{pH} 6$ (height is greater at the higher frequency), which corresponds to the cyclic voltammetric peak at $-\mathrm{r} .36 \mathrm{~V}$. NADP + and $\alpha-\mathrm{NAD}^{+}$exhibit similar a.c. peaks ; that for NADP+ is more positive than the NAD- peak while that for $\alpha-\mathrm{NAD}^{+}$is more negative. In the case of the second harmonic a.c. curves, in addition to those corresponding to peaks I and II, at high enough frequency $(500 \mathrm{~Hz})$, an additional derivative pattern is noticeable, whose potential $(-\mathrm{I} .32 \mathrm{~V})$ agrees with the fundamental a.c. and cyclic voltammetric patterns.

Exhaustive controlled-potential electrolysis of $\mathrm{NAD} \div$ (wave $\mathrm{I}$ potential) shows the absence of adenine reduction, as indicated by absorption spectra and the subsequent regeneration of $\mathrm{NAD}$ + during oxidation of the dimeric product. This result is in contrast to the observation of adenine reduction during uncontrolled-potential electrolysis. ${ }^{22}$ It is possible that in the latter case a specific chemical reductant for adenine was produced during the electrolysis.

Although all of the experimental evidence is consistent with reduction of the adenine moiety in $\mathrm{NAD}^{*}$, conclusive evidence is not available. The facts that $\mathrm{NAD}^{-}$largely exists in aqueous solution in a folded configuration, that at mercury electrodes the adsorption site in $\mathrm{NAD}^{+}$is the adenine moiety, and that $\mathrm{NAD}^{+}$undergoes reversible reduction in the adsorbed state, can be rationalized by a situation, in which folded $\mathrm{NAD}^{+}$is the adsorbed species at the solution|electrode interface with the more hydrophobic adenine ring'system facing the electrode and the more hydrophilic nicotinamide facing the solution with the electrontransfer process involving the passage of electrons from the electrode via the adenine to the pyridine ring.

Since adsorbed $\mathrm{NAD}^{+}$is initially reduced to a free radical, which dimerizes to an adsorbed dimer, it is difficult to use as a mechanismevaluative test the fact that adsorbed $\mathrm{NAD}^{+}$is reduced about $0 . I^{\prime} \mathrm{V}$ more easily than unadsorbed $N A D^{+} .{ }^{13}$

\section{Acknowledgment}

The authors thank the National Science Foundation, which helped support the work described. 


\section{References}

1 H.F. Fisher, E E. CoNi, B. Vexieslaxd and F H Westheimer, J. Biol. Chem. 202, 687 (1953)

9 MI E. Pullmax, A. Sizi Pietro and S.P Colowick, J. Biol Chem. 206, I29 (I954)

3 A I UNDERWOOD abd J.N. BURNETT, in Electroanalytical Chemistry, A.J. BARD (Editor), Marcel Dekher, New York (1972) Vol. 6, PP I-85

- P J. Elvixg, J E. O'Reilly and C O. Schuakel, in Methods of Brochemzcal Analyszs. D. Glick (Editor). Wiley-Interscience, New York (1973) Vol 21, pp. $28_{7}-{ }_{4} 6_{5}$

5 P J. Elvigg, C.O. Schinkel and K.S V. Saxthasam, Crit. Rez. Anal. Chem. 6, I (1976)

- P.J. Elvisg, in Topics in Bioelectrochemistry and Bioenergetics, G MIILazzo (Editor), John Wiley, London (1976) Vol. 1, pP I79-286

7 R.H. Sarbia and R.J. MIYxott, $J$ Am Chem Soc 95, 7470 (1973)

8 W.D Hamile, R.J. Pugmire and D MI Grañ, $J$ Am Chem Soc. 96, $288_{5}$ (I9T+)

9 J.W. WLib, B. Jaxik and P J Elvisg, J.Am. Chem Soc 95, 8495 (1973)

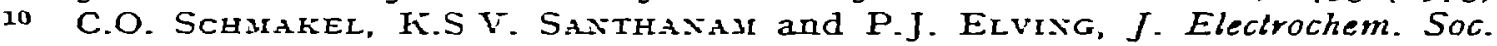
$121,3+5(1974)$

11 C O. Schmakel, K.S V. Saxthasay and P.J. Elvisg, J. Electrochem Soc. 121, $1033(1974)$

12 R.D. Braus, K S V. Santhanab and $\mathrm{P} J$ Elving, $J$. Am Chem. Soc. 97, 259I (1975)

13 C.O. Schmakel, K S V. Saxthaxim and P.J Elvixg, $J$ Am Chem. Soc. 97, 5083 (1975)

1: C O. Schuakel, Ph D Dissertation, The Unversity of Michigan, I97I

15 Mr.A. Jensex, Ph.D Disscrtation, The University of Michigan, 1977

$16 \mathrm{D} L$ Sinith and P.J. Elvixg, $J . A m$. Chem. Soc. 84, I 42 (1962)

17 D.L SMith and P J ElviNg, Anal Chem. 34, 930 (1962)

is G Drymurst and P.J. Elvisg, Talanta 16, 85j (I969)

19 IV S.V SAXThANAM and P J Elvixg, $J$. Am Chem. Soc. 96, 1653 (I974)

so K S V. Saxthanasi and P J. Elving, J. Am. Chem. Soc. 95, 5482 (r973)

21 B. Jasik and P.J. Elitisg, J. Am. Chem Soc 92, 235 (I970)

2 I.A. GorskayA A.V. Kotelxikova, FH F Sholis and S. YU. Drizovskaya, Brokhrmzya 30, 3I5 (I965)

23 A.V. Kotelinkova and V.V Solomatisa, Biokhimiya 30, Si6 (I965)

24 E.J. LAxD and A.J Swallow, Biochim Brophys Acta 162, 327 (1968)

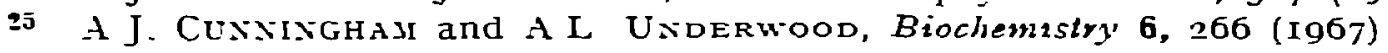

